\title{
LETTERS
}

\section{Using a whole-blood interferon- $\gamma$ assay to improve diagnosis of tuberculous pleural effusion}

\section{To the Editors:}

Tuberculous pleural effusion (TBPE) is treatable and curable, and hence it is important to establish the diagnosis and initiate treatment early. However, its diagnosis is not straightforward and depends on the demonstration of Mycobacterium tuberculosis in the pleural fluid or pleural biopsy specimen, or demonstration of caseating granulomas in the pleura. Due to the paucibacillary nature of this condition, sensitivity of pleural fluid smear for acid-fast bacilli is very low (0-1\%) [1]. Culture is relatively sensitive (12-50\%) [2], but is timeconsuming and requires standardised laboratories. The sensitivity of pleural biopsy ranges $39-80 \%$ [1, 3, 4] but this technique is invasive.

Current diagnostic options have several merits as well as limitations. Although no single test from the available battery may be completely accurate, an optimum combination of some of these tests may improve the diagnostic information [5-7]. We conducted this study to identify such a combination of tests for accurate diagnosis of TBPE. Specifically, we addressed two research questions. 1) What is the comparative diagnostic accuracy of the following five tests for the diagnosis of TBPE: the whole blood QuantiFERON ${ }_{\circledast}$-TB Gold In-Tube assay (QFTGIT) (Cellestis Ltd, Carnegie, Australia), adenosine deaminase (ADA) in pleural fluid, tuberculin sensitivity test (TST), PCR for mycobacteria and the lymphocyte/neutrophil ratio (L/N ratio) in pleural fluid? 2) Which strategy used to combine the results from these five tests can be most accurate in the diagnosis of TBPE?

This study included 151 consecutive patients (102 in the development sample and 49 in the validation sample) with exudative pleural effusion (defined using Light's criteria [8]) presenting at the Dept of Tuberculosis and Respiratory Diseases (Ganesh Shankar Vidyarthi Memorial Medical College (GSVMMC), Kanpur, India) between January 2006 and February 2007. Exclusion criteria were: age $<18$ yrs, pregnancy, positive HIV serology, on immunosuppressive treatment and previous history of anti-tuberculosis (TB) treatment. Median (interquartile range) age of the patients in the development sample was 47.5 (12) yrs; in the validation sample, it was 42 (23) yrs. The development and validation samples comprised $70.6 \%$ and $83.7 \%$ males, respectively. Detailed socio-demographic and clinical characteristics of the study subjects are provided in the online supplementary material. Informed consent was obtained from all the patients. The study was approved by the Ethics Committee, GSVMMC.
There were two main study groups in the development sample (fig. 1), TBPE and non-TBPE. The TBPE group included two categories of subjects. 1) Confirmed TBPE. Pleural fluid culture positive for $M$. tuberculosis and/or pleural biopsy histology showing a caseating granuloma. 2) Probable TBPE. Predominantly lymphocytic exudative pleural effusion with one of the following: pleural biopsy showing a chronic granulomatous inflammation without caseation; response to anti-TB treatment; or sputum culture positive for $M$. tuberculosis. These patients were classified as probable TBPE because granuloma without caseation can occur in conditions other than TB [9]. The non-TBPE group included two subgroups. 1) Other pleural effusion. Aetiology different from TB established by cytologic or histologic evaluation. 2) Undetermined pleural effusion. Pleural effusion that, by all conventional diagnostic methods, was of undetermined aetiology with no sign of active $\mathrm{TB}$ in the following 6 months.

Pleural fluid studies included cytologic examination, ZiehlNeelsen staining, ADA activity determination, PCR for TB bacilli and culture for M. tuberculosis. A pleural biopsy was also performed and the specimen was submitted for histopathologic examination. The QFT-GIT assay was conducted on $1 \mathrm{~mL}$ of venous blood incubated at $37^{\circ} \mathrm{C}$ for $16-24 \mathrm{~h}$. TST was considered positive if the size of the skin induration after 5 tuberculin units of RT-23 purified protein derivative (PPD) exceeded $10 \mathrm{~mm}$ after $72 \mathrm{~h}$ [10]. HIV testing was performed using ELISA. To identify a combination that would most parsimoniously and accurately classify the study subjects into TBPE and non-TBPE we used the classification and regression tree (CART) analysis (see supplementary material).

To directly compare the five tests with different outcome metrics, we first used receiver operating characteristic (ROC) curve analyses to dichotomise the results of continuous tests based on an optimum cut-off point. The area under the ROC curve for QFT-GIT and ADA was comparable and quite high (0.91 and 0.93 , respectively), while that for $\mathrm{L} / \mathrm{N}$ ratio was less (0.71). ROC analysis identified the optimum cut-off points for QFT-GIT, ADA and $\mathrm{L} / \mathrm{N}$ ratio as $0.38 \mathrm{IU} \cdot \mathrm{mL}^{-1}, 38 \mathrm{U} \cdot \mathrm{L}^{-1}$ and 3.0, respectively. The comparative performances for the five tests are shown in figure 1 and table 2 of the supplementary material. QFT-GIT and ADA had high sensitivity and specificity, L/N ratio had high sensitivity but poor specificity, PCR had high specificity and low sensitivity, while TST had, in general, lower sensitivity and specificity. The highest likelihood ratio of a positive test (LR+) was observed for PCR followed by QFT-GIT and ADA but the latter two tests also 
a)

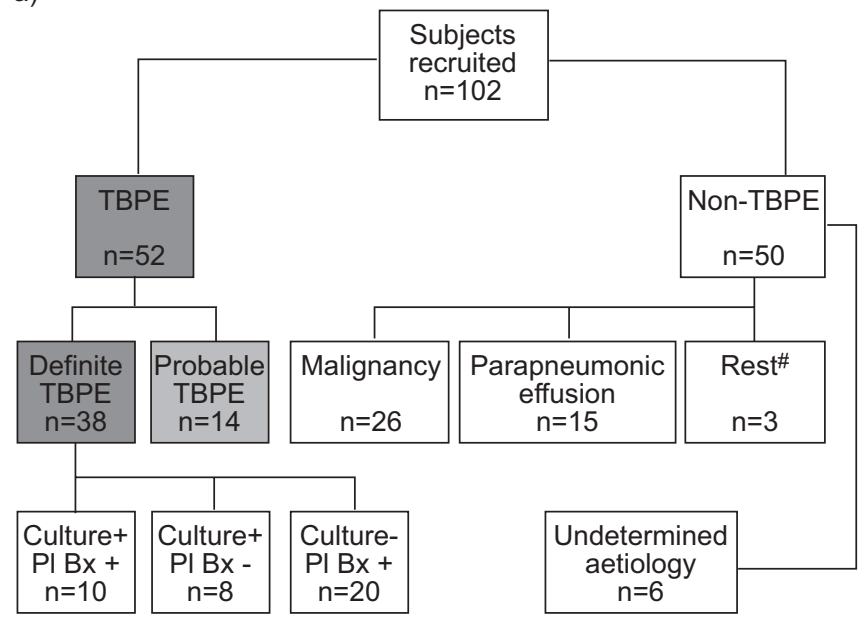

b)

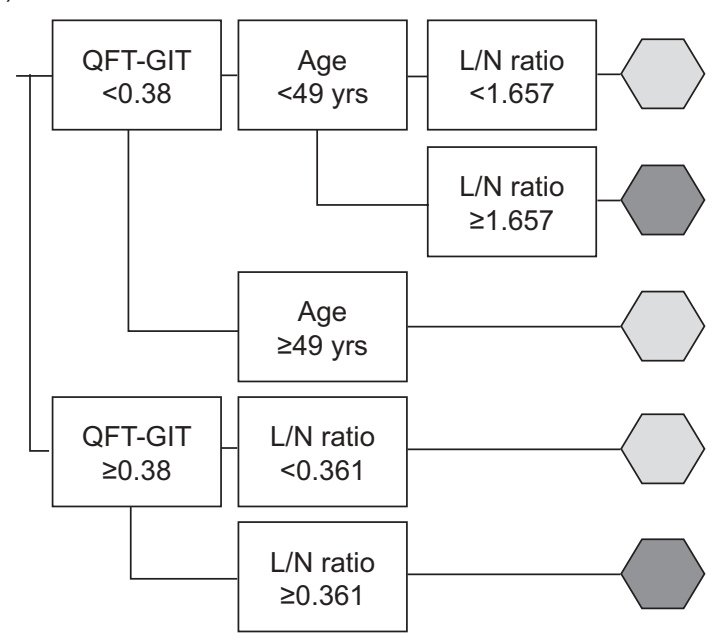

LR

0.00

1.65

0.00

0.00 c)

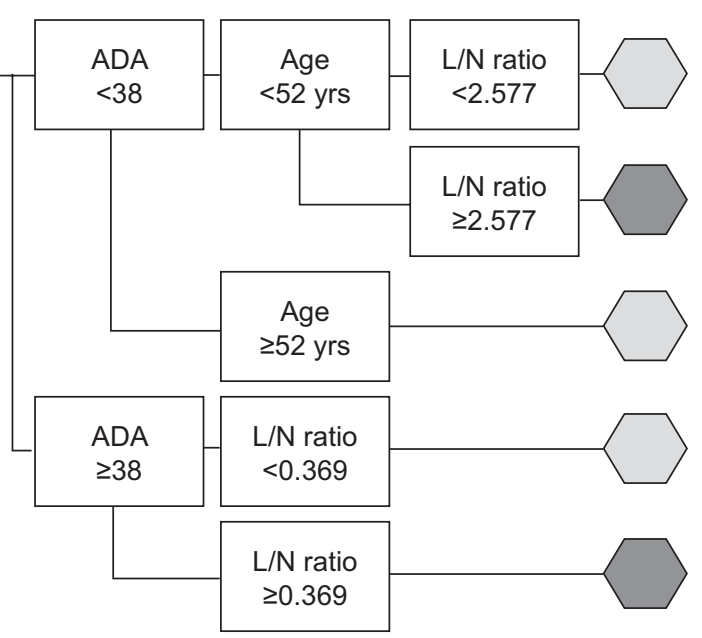

LR

0.00

2.24

0.00

0.00

17.4 d)

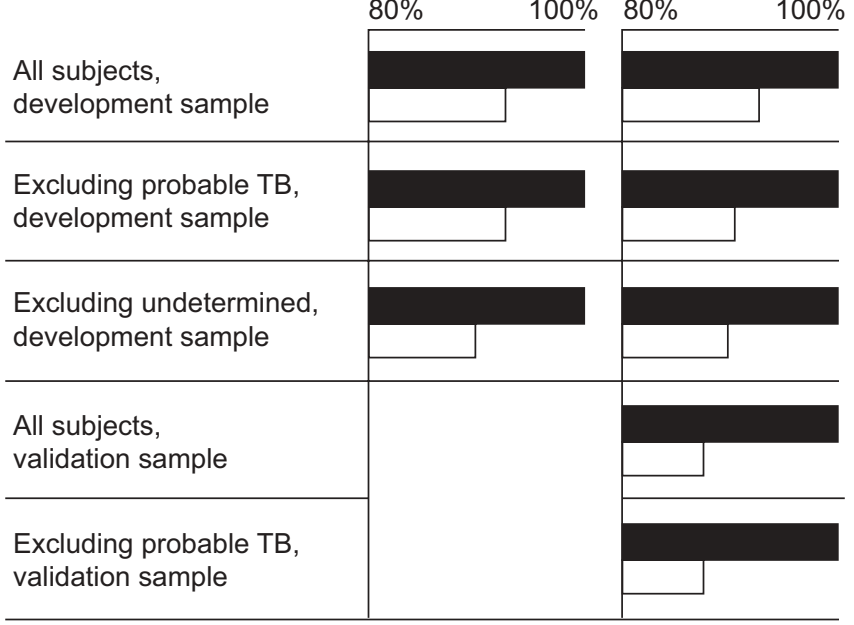

FIGURE 1. a) Characteristics of the study subjects. b) Results of the classification and regression tree (CART) analysis based on all five tests. The pruned final tree is shown as a series of nodal splits with the respective decision rules. The terminal nodes are shown as hexagons classified either as tuberculosis pleural effusion (TBPE; $\square$ ) or non-TBPE ( $\square$ ). Shown alongside each terminal node is the corresponding likelihood ratio (LR) for the diagnosis of TBPE. c) Results of CART analysis based on all tests except QuantiFERON ${ }_{\mathrm{w}}$-TB Gold In-Tube assay (QFT-GIT). d) Diagnostic performance of (b) and (c) in different subsets of the development and validation samples. In the development sample the diagnostic performance was evaluated in three scenarios: all subjects, subjects with probable TBPE excluded and subjects with undetermined TBPE excluded. In the validation sample, two scenarios were studied: all subjects and those excluding the probable TBPE. The bars show the sensitivity ( $\mathbf{\square})$ and specificity ( $\square$ ) for correct classification of study subjects. PI Bx: pleural biopsy. L/N: lymphocyte/neutrophil. ADA: adenosine deaminase. ${ }^{\#}$ : Two subjects with rheumatoid and one with uraemic aetiology

had the lowest likelihood ratio of a negative test (LR-). In contrast, L/N ratio and TST had poor discriminatory capacity as indicated by small LR+ and high LR- values.

In the CART analyses, we observed (fig. 1) that the pruned final tree contained three intermediate and five terminal nodes. In this tree, the first binary split was observed based on QFT-GIT test results, at a cut-off of $0.38 \mathrm{IU} \cdot \mathrm{mL}^{-1}$ based on the ROC analysis. The tree suggested that the diagnosis of TBPE can be considered for one of the following scenarios: 1) low IFN- $\gamma$ production $\left(<0.38 \mathrm{IU} \cdot \mathrm{mL}^{-1}\right)$, age $<49 \mathrm{yrs}$ and high $\mathrm{L} / \mathrm{N}$ ratio $(\geqslant 1.657)$; or 2$)$ high IFN- $\gamma$ production $\left(\geqslant 0.38 \mathrm{IU} \cdot \mathrm{mL}^{-1}\right)$ and moderately raised $\mathrm{L} / \mathrm{N}$ ratio $(\geqslant 0.369)$. The $\mathrm{LR}$ for diagnoses of TBPE in these two scenarios was 1.65 and 22.0, respectively, and indicated that different cut-off values for the $\mathrm{L} / \mathrm{N}$ ratio may be required to diagnose TBPE based on the result of the QFT-GIT. We evaluated the diagnostic performance in three subsets in the development sample as shown in figure 1. In all three subsets the sensitivity of the tree was $100 \%$ and specificity was at least $90 \%$. We refer to this tree as tree A hereafter.

Interestingly, tree A excluded ADA. We reasoned that this might be due to the identical diagnostic performance of QFTGIT and ADA in our dataset. To consider this possibility and 
also for the practical reason that many health centres are unlikely to possess the facilities needed to conduct the QFTGIT test, we repeated the CART analysis by excluding the QFT-GIT. We observed that the resulting tree was very similar to the tree A (fig. 1). Notably, the initial binary split was obtained based on ADA using a cut-off that was the same as that identified by the ROC analysis. This tree (referred hereafter to as tree B) suggested that the diagnosis of TBPE can be considered for one of the following two scenarios: 1) $\mathrm{ADA}<38 \mathrm{U} \cdot \mathrm{L}^{-1}$, age $<52 \mathrm{yrs}$, and $\mathrm{L} / \mathrm{N}$ ratio $\geqslant 2.577$ (LR 2.24); or 2) $\mathrm{ADA} \geqslant 38 \mathrm{U} \cdot \mathrm{L}^{-1}$ and $\mathrm{L} / \mathrm{N}$ ratio $\geqslant 0.369$ (LR 17.4). In different subsets of the development sample the diagnostic performance of trees A and B was on par (fig. 1).

Lastly, to ensure that our results from the development sample were not an artefact of data over-fitting, and also to examine the generalisability of our findings, we sought to determine whether the decision trees generated by CART algorithm could be used as a diagnostic aid in a separate sample of pleural effusion cases. The characteristics of the validation sample are described in the online supplementary material. Lack of financial support prevented us from conducting any more QFT-GIT tests and hence only tree B was used in the validation sample. In all subsets studied, tree B had 100\% sensitivity and $\geqslant 87.5 \%$ specificity. These findings indicate that the tree generated from the development sample could be successfully applied to the validation data set, thereby substantiating the utility of this approach in clinical practice. For example, it is recommended that lymphocytic preponderance combined with a pleural ADA level exceeding $40 \mathrm{U} \cdot \mathrm{L}^{-1}$ is highly indicative of TB [8]. Our findings, however, indicate that if pleural ADA levels are increased, then relatively small increases in the $\mathrm{L} / \mathrm{N}$ ratio may be sufficient for diagnosis of TBPE. In addition, our data point towards the importance of age in the diagnosis of TBPE in a developing country like India, where prevalence of TB is high. Most importantly, our study highlights the clinical utility of using a novel statistical tool (the CART algorithm) for the diagnosis of TBPE by combining the information content of available diagnostic options.

There are two additional key findings of our study. First, QFTGIT and ADA had equivalent and high diagnostic accuracy for TBPE compared to other tests, as observed by others [7, 11]. This is important from a public health viewpoint as the QFTGIT may not be available or affordable at many healthcare settings in developing countries. Secondly, and more importantly, we demonstrate that the TBPE test results can be combined for an improved diagnostic performance in line with a recent elegant review that identified the need for continued efforts for simple approaches to diagnosis of TBPE in the settings of high prevalence and low resources [12].

Studies of a similar nature, especially from areas with a low TB incidence, will be needed to endorse the generalisability of the diagnostic combinations and cut-offs identified herein. It is also unknown whether combination of the currently available and inexpensive biomarkers with INF- $\gamma$ assays based on the pleural fluid $[13,14]$ can further increase the diagnostic accuracy, and this will require additional studies.
S.K. Katiyar", A. Sampath ${ }^{*, \#}$, S. Bihari ${ }^{*}$, M. Mamtani ${ }^{\#, \oplus,+}$ and H. Kulkarni ${ }^{\#, \uparrow,+}$

*Dept of Tuberculosis and Respiratory Diseases, Ganesh Shankar Vidyarthi Memorial Medical College, Kanpur, "Lata Medical Research Foundation, Nagpur, India, "University of Texas Health Science Center at San Antonio, and ${ }^{+}$Veterans Administration Research Center for AIDS and HIV-1 Infection, South Texas Veterans Health Care System, San Antonio, TX, USA.

Correspondence: H. Kulkarni, 12023 Waterway Rdg, San Antonio, TX 78249, USA. E-mail: hemant_kulkarnius@yahoo.com

\section{REFERENCES}

1 Escudero Bueno C, Garcia Clemente M, Cuesta Castro B, et al. Cytologic and bacteriologic analysis of fluid and pleural biopsy specimens with Cope's needle. Study of 414 patients. Arch Intern Med 1990; 150: 1190-1194.

2 Chan $\mathrm{CH}$, Arnold M, Chan CY, et al. Clinical and pathological features of tuberculous pleural effusion and its long-term consequences. Respiration 1991; 58: 171-175.

3 Heidari B, Bijani K, Eissazadeh M, et al. Exudative pleural effusion: effectiveness of pleural fluid analysis and pleural biopsy. East Mediterr Health J 2007; 13: 765-773.

4 Gopi A, Madhavan SM, Sharma SK, et al. Diagnosis and treatment of tuberculous pleural effusion in 2006. Chest 2007; 131: 880-889.

5 Diacon AH, Van de Wal BW, Wyser C, et al. Diagnostic tools in tuberculous pleurisy: a direct comparative study. Eur Respir J 2003; 22: 589-591.

6 Menzies D, Pai M, Comstock G. Meta-analysis: new tests for the diagnosis of latent tuberculosis infection: areas of uncertainty and recommendations for research. Ann Intern Med 2007; 146: 340-354.

7 Hooper CE, Lee YC, Maskell NA. Interferon-gamma release assays for the diagnosis of TB pleural effusions: hype or real hope? Curr Opin Pulm Med 2009; 15: 358-365.

8 Porcel JM, Light RW. Diagnostic approach to pleural effusion in adults. Am Fam Physician 2006; 73: 1211-1220.

9 Jindal SK, Gupta D, Aggarwal AN. Sarcoidosis in developing countries. Curr Opin Pulm Med 2000; 6: 448-454.

10 Gothi GD, Chakraborty AK, Jayalakshmi MJ, et al. Use of 20 TU RT 23 and 5 TU Battery antigen for estimation of prevalence of non-specific tuberculin sensitivity. Indian J Med Res 1977; 66: 389-397.

11 Krenke R, Safianowska A, Paplinska M, et al. Pleural fluid adenosine deaminase and interferon gamma as diagnostic tools in tuberculosis pleurisy. J Physiol Pharmacol 2008; 59: Suppl. 6, 349-360.

12 Trajman A, Pai M, Dheda K, et al. Novel tests for diagnosing tuberculous pleural effusion: what works and what does not? Eur Respir J 2008; 31: 1098-1106.

13 Losi M, Bossink A, Codecasa L, et al. Use of a T-cell interferon- $\gamma$ release assay for the diagnosis of tuberculous pleurisy. Eur Respir J 2007; 30: 1173-1179.

14 Dheda K, Van-Zyl Smit RN, Sechi LA, et al. Clinical diagnostic utility of IP-10 and LAM antigen levels for the diagnosis of tuberculous pleural effusions in a high burden setting. PLoS One 2009; 4: e4689. 